\title{
LA FUNCIÓN DE LA RETROALIMENTACIÓN CORRECTIVA INDIRECTA EN LA REESCRITURA DE GÉNEROS ACADÉMICOS CON MODALIDAD NARRATIVA ELABORADOS POR ESTUDIANTES NOVATOS
}

\section{THE FUNCTION OF THE INDIRECT CORRECTIVE FEEDBACK IN THE ACADEMICS REWRITING WITH NARRATIVE MODALITY DEVELOPED BY NOVICE STUDENTS}

\author{
Steffanie Kloss Medina \\ Universidad Católica de la Santísima Concepción \\ Ciede-UCSC \\ skloss@ucsc.cl \\ Angie Quintanilla Espinoza \\ Universidad de Concepción \\ anquinta@udec.cl \\ Marcela Alexandre Moya \\ Pontificia Universidad Católica de Chile \\ m.alexandre@uc.cl
}

\begin{abstract}
Resumen:
La escritura es una habilidad compleja, más aún, si el estudiante no maneja la estructura genérica de las prácticas institucionalizadas. Por ello, el propósito de este estudio es indagar sobre los errores más frecuentes a nivel micro, macro y superestructural que cometen estudiantes novatos al escribir y reescribir un género académico de tipo narrativo, así como la efectividad de la retroalimentación correctiva indirecta en el proceso de reescritura. Para ello, se usó un método cuantitativo que permitió contabilizar los tipos de errores que comenten los sujetos en un primer y segundo escrito. Los hallazgos encontrados, señalan que los errores se presentan en los distintos niveles del discurso: microestructural, macroestructural y superestructural. Sin embargo, una vez que los textos son revisados y reescritos, solo los errores normativos persisten.
\end{abstract}

Palabras claves: Errores, Retroalimentación correctiva indirecta, Escritura, Reescritura.

\begin{abstract}
:
Writing is a complex skill, even more so, if the student does not handle the generic structure of institutionalized practices. Therefore, the purpose of this study is to investigate the most frequent errors that novice students make when writing and rewriting an academic genre of a narrative type at the micro, macro and superstructural level, as well as the effectiveness of indirect corrective feedback in the rewriting process. To do this, a quantitative method was used in order to count the types of errors that the subjects make in a first and second writing. The findings show that errors occur at different levels of discourse: microstructural, macrostructural and superstructural. However, just normative errors persist once the texts are revised and rewritten.
\end{abstract}


Artículo. Steffanie Kloss Medina, Angie Quintanilla Espinoza, Marcela Alexandre Moya. "La función de la retroalimentación correctiva indirecta en la reescritura de géneros académicos con modalidad narrativa elaborados por estudiantes novatos"

Key words: Errors, Indirect corrective feedback, Writing, Rewrite.

Recibido: 29 de septiembre de 2020

Aceptado: 10 de noviembre de 2020

\section{Introducción}

La escritura es una de las habilidades más complejas con las que se enfrenta un estudiante en el contexto universitario, consecuentemente, los profesores son llamados a monitorear este proceso ayudándolos a mejorar sus prácticas de redacción. Se ha investigado desde la línea de la alfabetización académica cómo el estudiante se inserta en la universidad (Carlino 13), se ha evaluado el contexto universitario y se han investigado las prácticas de escritura de los estudiantes (Ávila et al. 4) y también se ha estudiado la revisión de la escritura a través de estrategias de feedback y comentarios escritos. No obstante, los estudios en corrección de errores y estrategias de corrección se han dado mayoritariamente en lenguas extranjeras o en lengua materna, pero desde la perspectiva del feedback correctivo escrito a estructuras lingüísticas determinadas.

Ahora bien, para tener una visión global de la escritura es relevante identificarla como un proceso que implica tres etapas, a saber, la planificación, textualización y revisión (Flower y Hayes 369). Este estudio focalizará en la fase de textualización y revisión, puesto que se propone analizar la escritura de un texto a partir de borradores y el efecto que podría tener la retroalimentación correctiva indirecta en este proceso.

La incorporación de borradores es parte del proceso de escritura, pues el sujeto necesita volver sobre su texto e ir mejorándolo de manera constante. Sin embargo, cuando los escritores son aún nóveles no logran autorregular su proceso de escritura de manera inmediata, sino que necesitan de la guía de un escritor experto que corrija y guíe sus decisiones para elaborar un escrito de calidad.

De acuerdo con lo anterior, para establecer la calidad de un texto, Castelló (156) expone que deben tener en consideración siete tipos de dominios, a saber: conocimiento y profundización del tema, conocimiento lingüístico, es decir, cumplimiento de reglas 
léxicas, gramaticales y ortográficas, conocimiento de la situación retórica y, finalmente, conocimiento del género textual.

Estos antecedentes dan cuenta de la necesidad de diagnosticar cuáles son los problemas más frecuentes en la escritura de géneros académicos escritos por estudiantes novatos. De este modo, se podrán trabajar estrategias didácticas que den lineamientos a los docentes sobre cómo afrontar la producción escrita en contextos pedagógicos.

\section{Marco Teórico}

\subsection{Alfabetización académica}

La "alfabetización académica" ha sido definida como el "conjunto de nociones y estrategias necesarias para participar en la cultura discursiva de las disciplinas, así como en las actividades de producción y análisis de textos requeridas para aprender en la universidad" (Carlino Escribir 13). En este contexto, Hernández (57) señala que los estudiantes se acercan a la escritura a partir de su experiencia con los géneros discursivos frecuentes en su disciplina de estudio.

En general, cuando los estudiantes ingresan a la educación superior deben enfrentar un cambio de paradigma en la escritura, el cual va desde la reproducción de conocimiento a la transformación de conocimiento. De acuerdo con Errázuriz et al., es en este proceso es en el cual muchos estudiantes novatos se ven enfrentados a una real creación de textos (80). De este modo, Carlino (El proceso 322) ha determinado cuatro dificultades en alumnos universitarios novatos o inexpertos:

a. no considerar al lector: no se procesa el conocimiento, solo se reproduce.

b. desaprovechar la potencialidad epistémica de la escritura: no se cumple el propósito comunicativo del texto.

c. revisar solo la superficie del texto: no se profundiza en la corrección o mejora de ideas, sino que en revisar solo aspectos formales. 
Artículo. Steffanie Kloss Medina, Angie Quintanilla Espinoza, Marcela Alexandre Moya. "La función de la retroalimentación correctiva indirecta en la reescritura de géneros académicos con modalidad narrativa elaborados por estudiantes novatos"

d. postergar el momento de empezar a escribir: se recopila mucha información, pero se pospone el proceso de escritura, lo que lleva al escritor a centrarse en la prosa basada en el autor y no en sus propios pensamientos.

También es preciso considerar la falta de conocimiento de los géneros discursivos propios de cada disciplina y la situación comunicativa. Al respecto, también se señala que para superar estas dificultades es necesario que la comunidad educativa se involucre en el proceso (Carlino, El proceso 324). En palabras de Carlino el proceso de alfabetización académica "implica estar dispuesto a compartir con los alumnos la cultura académica que los profesores han adquirido como miembros de sus comunidades disciplinares" (91).

\subsection{Estudios en alfabetización académica en América Latina}

Los estudios en alfabetización académica o escritura en la universidad han proliferado en los últimos años, y en general, se observa una superioridad de artículos de tipo empírico por sobre los teóricos (Ortiz 35).

En una revisión bibliográfica realizada por Ortiz, se deja de manifiesto que los estudios en el área se concentran en dos enfoques: el enfoque cognitivo y el sociocognitivo. El enfoque cognitivo "encara la escritura como procesos mentales que son necesarios describir, en tanto tarea individual de resolución de problemas" (Ortiz, La escritura 21). El aporte de estos estudios se ha centrado en la creación de modelos estratégicos tendientes a apoyar a los estudiantes en los procesos mentales que debe llevar a cabo cuando escriben, incluyendo la organización, planificación y revisión del texto.

Por otro lado, el enfoque sociocognitivo señala que el contexto es un aspecto clave en el proceso de producción escrita. De este modo, los estudios que forman parte de este enfoque se centran en la escritura como práctica social y cultural (Calle-Arango y ÁvilaReyes 472).

Con respecto al objeto de estudio, las investigaciones publicadas en los últimos 20 años evidencian dos tendencias, a) una perspectiva pedagógica y ecléctica de la enseñanza 
de la lectura y la escritura y b) una perspectiva más bien lingüística y centrada en el discurso académico como producto.

En relación con los participantes, en general, los estudios de alfabetización son recurrentes en estudiantes de pregrado por sobre los estudiantes de posgrado, y especialmente en primer año. Algunas de estas investigaciones se han enfocado en determinar las dificultades que estos estudiantes tienen en la producción de textos, por ejemplo, carencia de bases para la escritura de textos argumentativos (Errázuriz et al., Diagnóstico 85), uso limitado de marcadores discursivos (Errázuriz 230), desconocimiento de procedimientos del ejercicio escrito (Córdova 26) y la reproducción imprecisa del contenido de textos citados (Hugo et al. 45), entre otros.

Finalmente, con respecto al diseño metodológico y las muestras, se observa una tendencia a utilizar muestras medianas o pequeñas. Mientras que se evidencia una disposición a los estudios de tipo cualitativo por sobre los cuantitativos. Algunos de estos estudios incluyen datos con respecto a las creencias y percepciones de los sujetos participantes, los cuales son obtenidos a partir de grupos focales y/o entrevistas.

\subsection{Niveles textuales: Micro, macro y superestructura}

El modelo de Van Dijk y Kintsch (52) presenta tres niveles de comprensión: la microestructura (nivel semántico), la macroestructura (nivel global) y la superestructura (estructura formal del texto).

La microestructura se refiere a los elementos locales y superficiales que hacen que un texto sea cohesivo y coherente, es decir, a las relaciones presentes en el texto a nivel morfológico, sintáctico y semántico. La microestructura incluye también mecanismos de cohesión en oraciones y párrafos (sustitución, elipsis, deixis textual, etc.) que permiten identificar relaciones y mantener la progresión temática al interior del texto.

Por otro lado, la macroestructura es de nivel más global (Van Dijk y Kintsch, Strategies 52); se define como un conjunto de macroproposiciones que describe un curso de eventos como un todo y que representa el sentido de un texto. En palabras de Van Dijk ( $L a$ ciencia 55), la macroestructura es "una representación de la estructura global de significado 
Artículo. Steffanie Kloss Medina, Angie Quintanilla Espinoza, Marcela Alexandre Moya. "La función de la retroalimentación correctiva indirecta en la reescritura de géneros académicos con modalidad narrativa elaborados por estudiantes novatos"

de un texto". Por esta razón, esta estructura puede ser entendida como el asunto o el tema del que trata un texto. La macroestructura se reconoce también como el mecanismo de coherencia textual que permite que un conjunto de oraciones pueda constituir un texto.

Finalmente, la superestructura corresponde a "un tipo de esquema abstracto que establece el orden global de un texto y que se compone de una serie de categorías, cuyas posibilidades de combinación se basan en reglas convencionales" (Van Dijk, La ciencia 144). Este esquema varía de acuerdo con el tipo de texto, por lo cual, en algunos casos, la superestructura permite realizar una configuración de los géneros discursivos. La superestructura constituye un esquema básico o esqueleto que establece el orden o la coordinación de las distintas partes que componen un texto. La figura 1 resume los componentes que forman parte la estructura textual.

\begin{tabular}{|c|c|c|}
\hline \multicolumn{3}{|c|}{ Estructuras textuales } \\
\hline MICROESTRUCTURA & MACROESTRUCTURA & SUPERESTRUCTURA \\
\hline Nivel Sintáctico & Nivel semántico & Nivel discursivo \\
\hline Microproposiciones: palabra & $\begin{array}{l}\text { Macroproposiciones: } \\
\text { organización, estructura } \\
\text { local. }\end{array}$ & $\begin{array}{l}\text { Género discursivo: } \\
\text { Estructura global }\end{array}$ \\
\hline \multicolumn{3}{|l|}{ CATEGORÍAS } \\
\hline $\begin{array}{l}\text { Ortografía literal, acentual y } \\
\text { puntual. } \\
\text { Uso de preposiciones }\end{array}$ & $\begin{array}{l}\text { Concordancia gramatical de } \\
\text { género y número. } \\
\text { Marcadores discursivos. } \\
\text { Correferencia. } \\
\text { Orden de palabras }\end{array}$ & $\begin{array}{l}\text { Características del género } \\
\text { discursivo académico } \\
\text { profesional: } \\
\text { - Estructura. } \\
\text {-Propósito comunicativo. }\end{array}$ \\
\hline
\end{tabular}

Figura 1. Análisis de errores y estructuras textuales (Kloss 65)

Entender el discurso de acuerdo con estos tres niveles permite conocer su estructura y los elementos circunscritos en cada nivel. En el ámbito de la superestructura se organiza el género y textura discursiva. Para efectos de esta investigación, es pertinente caracterizar la narración. Van Dijk (La ciencia 156) es uno de los primeros investigadores en desarrollar propuestas en torno a la superestructura narrativa, proponiendo un diagrama arbóreo (ver figura 2). 


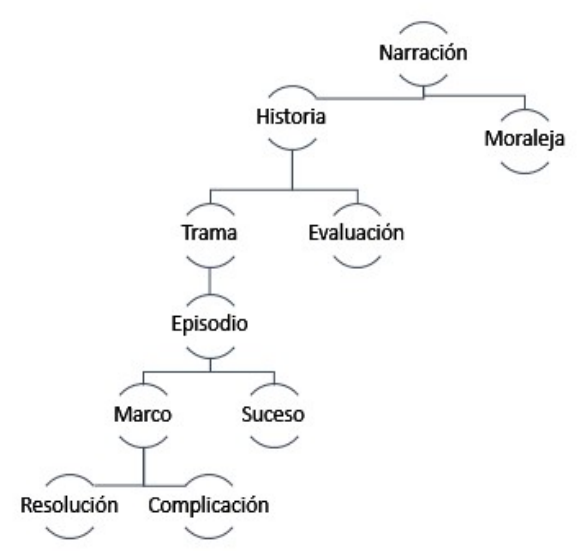

Figura 2. Diagrama de la superestructura narrativa

Por otro lado, Adam (62) formula una nueva secuencia para la superestructura narrativa (ver figura 3) a partir de la propuesta inicial de Van Dijk.

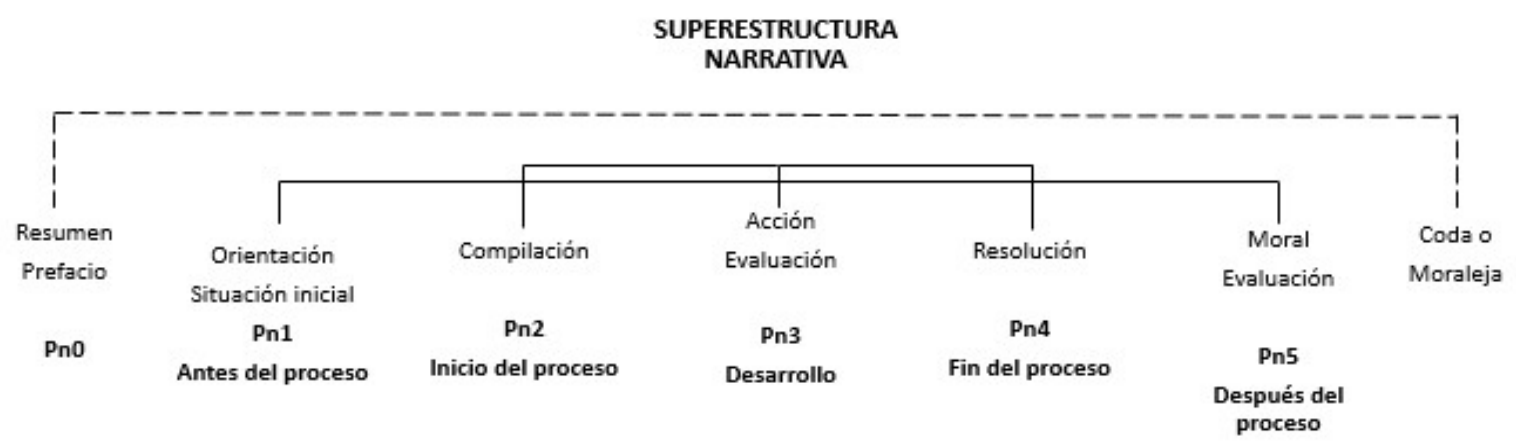

Figura 3. Secuencia narrativa

Este esquema narrativo, permite distinguir seis elementos básicos presentes en toda narración: temporalidad o sucesión de eventos, unidad temática (sujeto-actor), transformación o cambio de los estados, unidad de acción (situación inicial - situación final) y causalidad entre los acontecimientos.

\subsection{Errores}

Al referirse a errores es necesario realizar una distinción entre las "faltas" y los “errores". De acuerdo con Brown, una falta se refiere a "un fracaso para utilizar un sistema conocido correctamente" mientras que un error dice relación con "una notoria desviación 
Artículo. Steffanie Kloss Medina, Angie Quintanilla Espinoza, Marcela Alexandre Moya. "La función de la retroalimentación correctiva indirecta en la reescritura de géneros académicos con modalidad narrativa elaborados por estudiantes novatos"

de la gramática adulta de un hablante nativo" (Brown 205). Con respecto a esta distinción Kotz y Ferreira (221) señalan que los errores del aprendiente se deben a la "falta de competencia lingüística" y que las faltas, por otro lado, "son producto del cansancio, del estrés o de la falta de concentración".

Es necesario señalar que las faltas no requieren de un tratamiento especial por parte del profesor, puesto que en estos casos el estudiante posee la competencia lingüística para reconocer la falta y autocorregirla. Sin embargo, en el caso de los errores no ocurre lo mismo. Los errores tienden a ser sistemáticos y no reconocidos por el aprendiente, razón por la cual no pueden ser autocorregidos (Corder 152).

\subsection{Clasificación de errores}

Vázquez (1999) propone una clasificación de errores sobre la base de seis criterios: lingüístico, etiológico, comunicativo, pedagógico, pragmático y cultural. La figura 4 presenta una descripción de esta taxonomía.

\begin{tabular}{|l|l|}
\hline \multicolumn{1}{|c|}{ Criterio } & \multicolumn{1}{c|}{ Tipos de errores } \\
\hline Lingüístico & $\begin{array}{l}\text { Errores de adición } \\
\text { Errores de omisión } \\
\text { Errores de yuxtaposición } \\
\text { Errores de falsa colocación } \\
\text { Errores de falsa selección }\end{array}$ \\
\hline Etiológico & $\begin{array}{l}\text { Errores interlinguales } \\
\text { Errores intralinguales } \\
\text { Errores de simplificación }\end{array}$ \\
\hline Comunicativo & $\begin{array}{l}\text { Errores de ambigüedad } \\
\text { Errores irritantes } \\
\text { Errores estigmatizantes } \\
\text { Errores de falta de pertinencia }\end{array}$ \\
\hline
\end{tabular}




\begin{tabular}{|l|l|} 
Pedagógico & Errores inducidos vs. creativos \\
& Errores transitorios vs. permanentes \\
& Errores fosilizados vs. fosilizables \\
& Errores individuales vs. colectivos \\
& Errores residuales vs. actuales \\
& Errores congruentes vs. idiosincrásicos \\
& Errores de producción oral vs. escrita \\
& Errores globales vs. locales
\end{tabular}

Figura 4. Taxonomía de errores

\subsection{Errores más frecuentes en español}

Reyes presenta los errores gramaticales más frecuentes en prosa universitaria y textos de prensa en español (89-97). Estos se agrupan como sigue:

1. Concordancia: género y número.

1. Determinativos: partitivos, ordinales y distributivo.

2. La apócope de primero.

3. Verbos pronominales.

4. Leísmo y laísmo.

5. Uso de gerundio: tiempo y función.

6. Oraciones interrogativas indirectas.

7. Pronombres relativos.

8. Verbo impersonal haber.

9. Forma verbal en -ría.

10. Comparativos.

11. Oraciones impersonales con se.

12. Colocación del pronombre átono. 
Artículo. Steffanie Kloss Medina, Angie Quintanilla Espinoza, Marcela Alexandre Moya. "La función de la retroalimentación correctiva indirecta en la reescritura de géneros académicos con modalidad narrativa elaborados por estudiantes novatos"

\subsection{Corrección de errores}

Cassany propone que la corrección de errores consta de 2 pasos básicos: buscar defectos, errores o imperfecciones y revisar o reformular dichos errores (Cassany 25).

La corrección de errores tiene los siguientes objetivos (Cassany, Reparar 30):

a. informar al alumno sobre su texto a través de una impresión global, información de tipo léxica, gramatical u otra, e identificación de los errores.

b. conseguir que el alumno modifique su texto a partir de indicaciones generales para mejorar y corregir las faltas.

c. lograr que el alumno mejore su escritura, aprenda de los errores que ha cometido y aprenda gramática y ortografía.

d. cambiar el comportamiento del alumno a través del aprendizaje de técnicas de redacción.

Del mismo modo, este autor (Cassany, Reparar 31) plantea que los errores que deben ser corregidos son básicamente aquellos relacionados con lo siguiente: normativa (ortografía, morfología y sintaxis y léxico), cohesión (puntuación, nexos y anáforas), coherencia (selección de la información, progresión de la información, estructura del texto y estructura del párrafo), adecuación (selección de la variedad, selección del registro, fórmulas y giros estilísticos) y otros aspectos como la disposición del texto en la hoja, tipografía, estilística y variación (léxica o sintáctica).

\subsection{Corrección de la escritura y retroalimentación}

La retroalimentación correctiva $(\mathrm{RC})$ surge como evidencia negativa, a partir de la exposición a una lengua distinta a la materna, es decir, un estudiante que se expone al aprendizaje de una L2. Cabe mencionar que esa evidencia en el contexto de práctica puede ser positiva o negativa; la evidencia positiva proporciona información con respecto a lo que es posible y gramaticalmente aceptable, mientras que la evidencia negativa proporciona 
información en torno a lo que es inaceptable en la L2. En este contexto, Chaudron (429) utiliza el término "corrección de errores" para referirse a movimientos correctivos que conducen a la reparación del error. Además, introduce una de las primeras técnicas correctivas, pues señala que la RC corresponde a la reacción del profesor, quien modifica, desaprueba o demanda mejorar algún error cometido por un estudiante. Por su parte, Ellis (99) concuerda con que el feedback correctivo (FC) representa una respuesta frente a un enunciado inexacto del estudiante, apreciación que también es compartida por Van Beuningen (5) quien destaca que la importancia del FC radica en su propiedad de inducir la focalización de la atención de los estudiantes en la forma, por lo que según Sheen el FC sería "una invitación del profesor a los estudiantes a prestar atención a la precisión gramatical de algo que han dicho o escrito" (Sheen 1).

Si bien las investigaciones en la RC se inician en el aprendizaje de una segunda lengua o lengua extranjera, el mejoramiento de los procesos de escritura también es un tema relevante en una lengua materna porque es un proceso que necesita varias microhabilidades para que resulte de manera exitosa. En ese sentido, Cassany (Reparar 71) en el texto Reparar la Escritura, entrega indicios de cómo el docente de L1 debe corregir a sus estudiantes, mediante la focalización de los errores, cómo marcarlos en un texto, qué color de lápiz utilizar, pero también explica que esto aún no es suficiente para determinar o combinar estrategias desde este ámbito. De acuerdo con lo anterior, la RC escrita es pertinente, ya que la implementación de la retroalimentación es apropiada para que el docente apoye el proceso de enseñanza de la escritura. Además, proporciona al estudiante andamiajes de las formas gramaticales y discursivas que necesita para dar coherencia y cohesión a los textos en un contexto comunicativo donde no se memoricen las formas, sino que se modelen a través del aprendizaje de la escritura y la corrección de errores.

\subsection{Clasificación de retroalimentación correctiva escrita (RCE)}

Para el tratamiento de los errores en la producción escrita, Ellis (A typology 98) describe y ejemplifica tipologías de estrategias de RCE (ver figura 5). 
Artículo. Steffanie Kloss Medina, Angie Quintanilla Espinoza, Marcela Alexandre Moya. "La función de la retroalimentación correctiva indirecta en la reescritura de géneros académicos con modalidad narrativa elaborados por estudiantes novatos"

\begin{tabular}{|c|c|}
\hline $\begin{array}{l}\text { Estrategias para proveer } \\
\text { RCE }\end{array}$ & Descripción \\
\hline 1. RCE directa & El profesor provee al estudiante la forma correcta. \\
\hline 2. RCE indirecta & $\begin{array}{l}\text { El profesor indica al estudiante que se ha cometido un error, pero } \\
\text { no provee la forma correcta. }\end{array}$ \\
\hline $\begin{array}{l}\text { a. Indicación y } \\
\text { localización del } \\
\text { error }\end{array}$ & $\begin{array}{l}\text { Este error puede ser subrayado o marcado para mostrar al } \\
\text { estudiante la ubicación del error. }\end{array}$ \\
\hline b. Solo indicación & $\begin{array}{l}\text { Solo se indica en el margen que se ha cometido un error en una } \\
\text { línea del texto. }\end{array}$ \\
\hline $\begin{array}{l}\text { 3. } \mathrm{RCE} \\
\text { metalingüística }\end{array}$ & $\begin{array}{l}\text { El profesor entrega al alumno algún tipo de clave metalingüística } \\
\text { con referencia a la naturaleza del error. }\end{array}$ \\
\hline $\begin{array}{l}\text { a. Uso de código de } \\
\text { error }\end{array}$ & El profesor escribe códigos en el margen. \\
\hline $\begin{array}{l}\text { b. Breve descripción } \\
\text { gramatical }\end{array}$ & $\begin{array}{l}\text { El profesor enumera los errores en el texto y escribe (al final de la } \\
\text { página) una descripción gramatical para cada error numerado. }\end{array}$ \\
\hline $\begin{array}{l}\text { 4. El foco de la } \\
\text { retroalimentación }\end{array}$ & $\begin{array}{l}\text { Corresponde al enfoque del profesor con respecto a la corrección } \\
\text { de todos (o la mayoría) de los errores o a la selección de uno o dos } \\
\text { tipos de errores específicos para corregir. }\end{array}$ \\
\hline a. RCE no focalizada & La RCE no focalizada es extensiva. \\
\hline b. RCE focalizada & La RCE focalizada es intensiva. \\
\hline $\begin{array}{l}\text { 5. Retroalimentación } \\
\text { electrónica }\end{array}$ & $\begin{array}{l}\text { El profesor indica un error y provee un hipervínculo a un archivo } \\
\text { de concordancia que muestra ejemplos del uso correcto. }\end{array}$ \\
\hline 6. Reformulación & $\begin{array}{l}\text { Consiste en la reconstrucción completa del texto por parte de un } \\
\text { hablante nativo. El propósito es hacer que el lenguaje utilizado se } \\
\text { parezca lo más posible a la lengua meta sin perder el contenido del } \\
\text { texto original. }\end{array}$ \\
\hline
\end{tabular}

Figura 5. Tipología de estrategias de retroalimentación correctiva escrita de Ellis

\section{Método}

Con respecto a la metodología, esta investigación se orienta a los llamados métodos cuantitativos, ya que busca en este orden describir a partir de la medición y valorización de 
criterios lingüísticos con dimensiones específicas lo que significa que un texto sea de calidad, de acuerdo con los requerimientos que implica desarrollar la escritura en un contexto universitario. Sobre esta base, surge como objetivo general para guiar este estudio indagar cuáles los errores más frecuentes a nivel micro, macro y superestructural que cometen estudiantes novatos al escribir y reescribir un género académico de tipo narrativo, así como la efectividad de la retroalimentación correctiva indirecta en el proceso de reescritura.

Esta investigación es de carácter no experimental, por lo tanto, no considera la variación de las variables independientes. Su propósito es levantar una observación descriptiva del fenómeno como tal. El alcance de este estudio es longitudinal, puesto que considera observar un mismo grupo de sujetos en dos procesos lingüísticos consecutivos; elaboración de un texto y reescritura de este. Entre un momento y otro se definió una instancia de evaluación con el fin de identificar los errores frecuentes para distinguir cuáles son los que se generan en la escritura primera y cuáles son los que se dan en la instancia de la reescritura.

Con respecto a la población y muestra se definió trabajar con 42 estudiantes de primer año, que cursan una asignatura de escritura y que pertenecen a las carreras de nutrición y dietética, periodismo y derecho de una universidad chilena. El criterio de selección fue estructurado de acuerdo con el resultado de aprendizaje del curso de escritura, el que estaba relacionado con la elaboración de textos académicos, según la intención comunicativa. Ello con el propósito de vincular a los estudiantes con sus respectivas disciplinas y con las destrezas necesarias para redactar los géneros de su especialidad.

\subsection{Descripción de la tarea}

Los estudiantes recibieron como estímulo un texto narrativo titulado Por qué escribo. La función de este escrito fue mostrar cómo puede forjarse el gusto por la escritura y por qué es relevante manejar esta práctica como habilidad superior y necesaria para desarrollarse en la universidad. Posteriormente, se les entregaron las instrucciones para ejecutar la tarea con la siguiente consigna: Escribir consiste en desarrollar un ejercicio 
Artículo. Steffanie Kloss Medina, Angie Quintanilla Espinoza, Marcela Alexandre Moya. "La función de la retroalimentación correctiva indirecta en la reescritura de géneros académicos con modalidad narrativa elaborados por estudiantes novatos"

cognitivo que necesita, entre otras cosas, de la motivación. Escribimos porque queremos hacerlo, porque suponemos que la escritura nos ayuda a participar de la vida social y, también, académica y profesional. En este ejercicio se les propuso a los sujetos escribir un texto de carácter expresivo en que se comunique la motivación que tienen para escribir y relacionar este aspecto con la selección de su carrera. La tarea de escritura se organizó de acuerdo con los siguientes criterios:

a. Tema: Motivación para la escritura.

b. Propósito: Expresivo.

c. Extensión: 400 a 600 palabras.

\subsection{Escritura y reescritura de la tarea}

Los estudiantes, luego de una sesión de escritura tipo taller, en la que leyeron sus trabajos y escucharon la opinión de sus tutores y compañeros, entregaron el primer borrador de su texto. Al día siguiente, este era revisado por dos tutores y la docente a cargo del curso para calibrar la corrección a través de la rúbrica de evaluación. Este grupo de expertos revisaba cada uno de los textos con una retroalimentación correctiva indirecta en los diferentes niveles textuales: Micro, macro y superestructural. A continuación, se presentan ejemplos de comentarios escritos entregados a los estudiantes de acuerdo con los tipos de errores encontrados:

\begin{tabular}{|l|l|l|}
\hline Corpus & Ejemplo de error & Retroalimentación correctiva indirecta \\
\hline $\begin{array}{l}\text { S1-PER- } \\
\text { ESC1. }\end{array}$ & $\begin{array}{l}\text { ¿Qué me llevó a elegir mi carrera? Largo } \\
\text { proceso". }\end{array}$ & $\begin{array}{l}\text { Recuerde que el texto narrativo tiene } \\
\text { una estructura textual, entonces el título } \\
\text { es relevante para clarificar el contenido } \\
\text { del relato y las características narrativas. } \\
\text { Para ello, es importante que piense en } \\
\text { qué quiere abordar en su escrito, cómo } \\
\text { organiza la información para que sea } \\
\text { legible. Para lograrlo, es importante que } \\
\text { si plantea preguntas retóricas, estas } \\
\text { efectivamente inviten al lector a a } \\
\text { comprender su relato y a disfrutarlo. }\end{array}$ \\
\hline
\end{tabular}


Este tipo de retroalimentación era recurrente a nivel superestructural. La retroalimentación correctiva indirecta permite hacer notar al sujeto qué errores tiene, para que este sea capaz de pensar en qué componentes necesita para que su título sea redactado de manera atractiva y organizada, puesto que no recibe una corrección explícita.

\begin{tabular}{|c|c|c|}
\hline Corpus & Ejemplo de error & Retroalimentación correctiva indirecta \\
\hline $\begin{array}{l}\text { S1-DER- } \\
\text { ESC1. }\end{array}$ & $\begin{array}{l}\text { "(...) cuando estaba comenzando primero } \\
\text { medio, pero generalmente cuando uno } \\
\text { piensa algo demasiado } \\
\text { le va encontrando peros, y así fue como } \\
\text { pasé los siguientes } 3 \text { años, cambiando } \\
\text { todos los meses la carrera que iba a } \\
\text { estudiar, intentando explorar mis } \\
\text { habilidades, mis intereses, pero eso solo } \\
\text { me llevaba a tener cada vez más opciones } \\
\text { porque me considero una persona muy } \\
\text { multifacética, el no tenerlo decidido no } \\
\text { me preocupaba tanto porque todavía me } \\
\text { quedaba tiempo, pero cuando (...)" }\end{array}$ & $\begin{array}{l}\text { El uso de marcadores permite conectar } \\
\text { las ideas de su texto. La conexión 'pero' } \\
\text { indica oposición de ideas. Sobre esa } \\
\text { base ¿eso quiere expresar? } \\
\text { Además, sugiero diversificar el uso de } \\
\text { conexiones de oposición para mejorar la } \\
\text { organización de su escrito. }\end{array}$ \\
\hline
\end{tabular}

Tabla 2. Error en la macroestructura

La retroalimentación correctiva de tipo indirecta permite hacer que el escritor note los aspectos mejorables en su escrito. El ejemplo anterior muestra la RCE que se le dio a un sujeto en torno al uso de marcadores discursivos y se relevó la función de estas partículas discursivas para la construcción de un texto cohesivo.

\begin{tabular}{|l|l|l|}
\hline Corpus & Ejemplo de error & \multicolumn{1}{|l|}{$\begin{array}{l}\text { Retroalimentación correctiva } \\
\text { indirecta }\end{array}$} \\
\hline $\begin{array}{l}\text { S1-NUT- } \\
\text { ESC1. }\end{array}$ & $\begin{array}{l}\text { La vida me había quitado algo, muy } \\
\text { importante al decir verdad. Me robo } \\
\text { mi imaginacion y mi inocencia. }\end{array}$ & $\begin{array}{l}\text { Usó dos palabras agudas que necesitan } \\
\text { ser tildadas. Recuerde que todas las } \\
\text { palabras que se consideren de ese tipo y } \\
\text { que terminen en 'n, s o vocal' se tildan } \\
\text { en la última sílaba. }\end{array}$ \\
\hline
\end{tabular}

Tabla 3. Error en la microestructura

Si bien es cierto la ortografía se considera el último aspecto que debe corregirse en un escrito (Cassany Reparar 13), los textos analizados presentaron recurrencia en este tipo 
Artículo. Steffanie Kloss Medina, Angie Quintanilla Espinoza, Marcela Alexandre Moya. "La función de la retroalimentación correctiva indirecta en la reescritura de géneros académicos con modalidad narrativa elaborados por estudiantes novatos"

de error. La ortografía puntual, literal y acentual, manifiestan dificultades porque los sujetos manejan escasamente las reglas gramaticales y cuando reescriben su texto corrigen los errores retroalimentados de manera indirecta, pero no son capaces de integrar esa regla y, por lo tanto, cometen nuevos errores en este ámbito.

\subsection{Descripción del instrumento de evaluación y sistema de medición de errores}

Para revisar los textos se utilizó una rúbrica con 10 descriptores. En primer lugar, se consideró la superestructura, sobre esa base se evaluó el componente narrativo, luego la estructura, el tema y, finalmente, el propósito. Estos cuatro aspectos se calificaron de manera dicotómica, es decir, 0 para la ausencia de error y 1 para delimitar problema o error es ese ámbito.

En segundo lugar, se trabajó con la macroestructura. Allí se contabilizaron los errores totales de cada texto. Para ello se corrigió lo siguiente: coherencia, cohesión y vocabulario. Este nivel da cuenta de la organización local y global del escrito porque remite al dominio lingüístico de los alumnos en relación con el uso de palabras adecuadas al mensaje, pero también a la variedad lingüística para evitar la repetición de palabras. Asimismo, este nivel precisa el uso de marcadores discursivos, pues a través del análisis, se permite diagnosticar la conexión de las ideas que se marcan en el texto.

En tercer lugar, se evaluó el nivel microestructural. En este se contabilizaron todos los errores de ortografía literal de acuerdo con el uso de mayúscula y minúscula, así como el uso adecuado de letras c, s; v, b. Luego, la ortografía acentual, según las reglas de tildación y, para finalizar, la ortografía puntual, que permitió diagnosticar el uso de la coma, punto seguido, aparte y final.

\subsection{Corpus}

El corpus lingüístico remite a una recopilación de textos que se conciben necesariamente en un soporte electrónico (Cruz Piñol 26) y según ciertos criterios objetivos de selección de textos. Para efectos de esta investigación, se seleccionaron 84 textos 
escritos por estudiantes de primer año de una universidad chilena en las carreras de nutrición y dietética, periodismo y derecho.

Los 84 textos corresponden a 42 escrituras y 42 reescrituras de una composición con modalidad narrativa. Este género discursivo es un escrito que tiene como finalidad motivar a los estudiantes a escribir, por ello se trabaja con la estructura narrativa de inicio, complicación, desarrollo y cierre con una temática definida, que hacía referencia a por qué es importante desarrollar la escritura en la universidad y cómo se relaciona esta actividad con la carrera que eligieron estudiar. La extensión del escrito fluctuaba entre 400 y 600 palabras.

Para organizar el corpus, se etiquetaron los textos de la siguiente manera: Se asignó un número al sujeto ( $\mathrm{S} 1$ ), se escribió la inicial de la carrera que cursa (PER-periodismo), se agregó si el escrito correspondía a escritura (ESC1) o reescritura (ESC2) y finalmente se asignó una etiqueta S1-PER-ESC1.

\section{Resultados}

Los resultados de la aplicación de este estudio dan cuenta del proceso de escritura que llevan a cabo estudiantes novatos, a través del diagnóstico de cuáles son los errores más frecuentes y cómo opera la retroalimentación correctiva indirecta emitida por los sujetos revisores en la superación de esos errores. El análisis del corpus determina que los errores que cometen con mayor frecuencia se dan en la ortografía puntual, tanto en el primer como segundo escrito. Sin embargo, en la reescritura hay una disminución significativa de los errores.

Es decir, al analizar los datos de frecuencia de los errores del primer borrador escrito por los estudiantes de las tres carreras mencionadas, se puede observar que el rango de errores en la superestructura fluctúa entre 16 y 29. Este nivel se analizó considerando los valores dicotómicos 0 y 1 , se etiquetó con 0 cuando el componente narrativo, la estructura, la organización del tema y el propósito eran correctos, mientras que se valoró con 1 la ausencia de estos criterios. En cuanto a la reescritura, podemos percibir que, la cantidad de 
Artículo. Steffanie Kloss Medina, Angie Quintanilla Espinoza, Marcela Alexandre Moya. "La función de la retroalimentación correctiva indirecta en la reescritura de géneros académicos con modalidad narrativa elaborados por estudiantes novatos"

errores fluctuó entre 0 y 4 formas erróneas. De manera detallada se observa en el gráfico $\mathrm{N}^{\circ}$ 1 que la mayor complicación se presentó en la estructura narrativa.

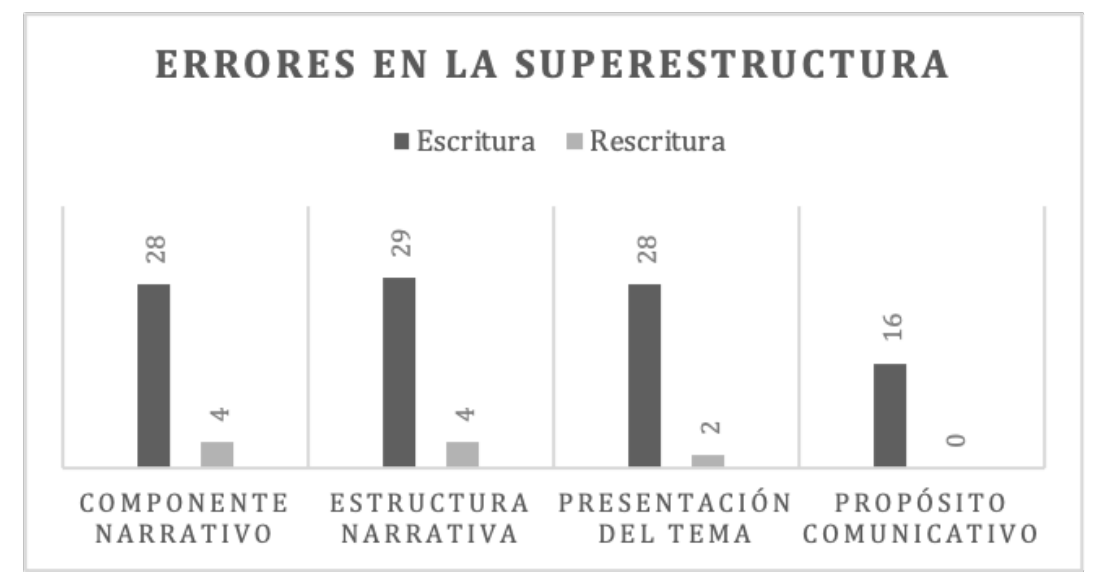

Gráfico 1. Errores en la superestructura

A continuación, se muestran los errores presentes en la macroestructura, estos se clasificaron de acuerdo con su presencia en el texto. La mayor cantidad de errores se presentó en el vocabulario, con un total de 105 errores; seguido por 83 en la cohesión y 50 en la coherencia. Los errores disminuyeron considerablemente en la reescritura, por lo que el efecto correctivo provocó que los jóvenes disminuyeran sus errores.

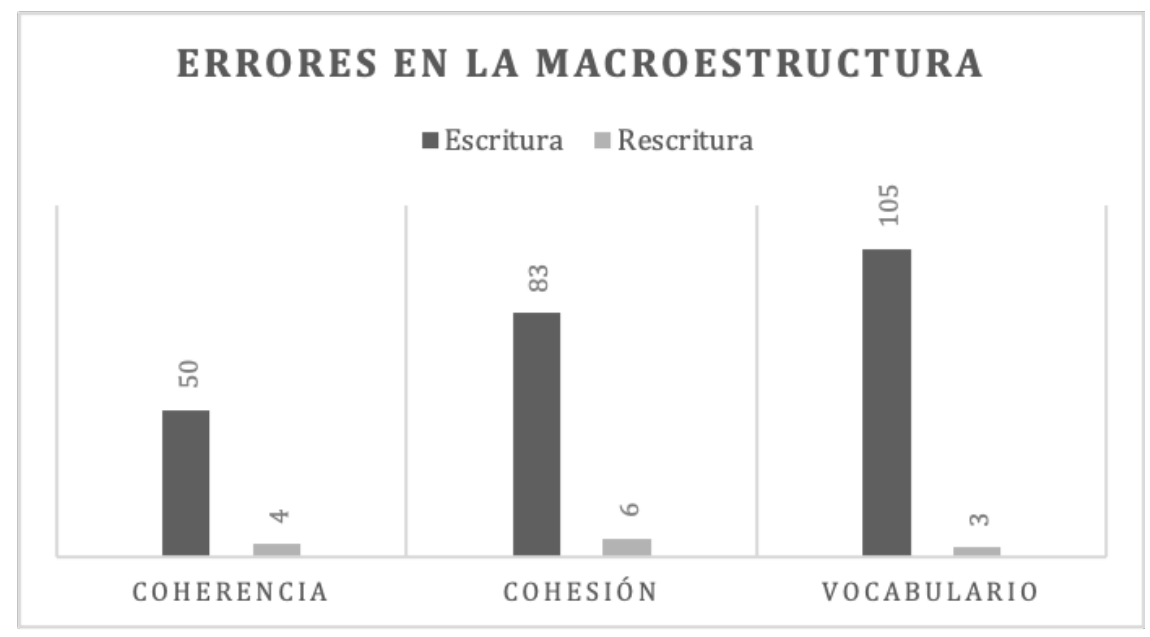

Gráfico 2. Errores en la microestructura 
Los resultados en torno a la microestructura ponen de manifiesto la concentración mayor de errores en el corpus. El gráfico 3 muestra en detalle la cantidad de errores en la primera y segunda escritura, en este aspecto, la mayor complejidad estuvo situada en la ortografía puntual.

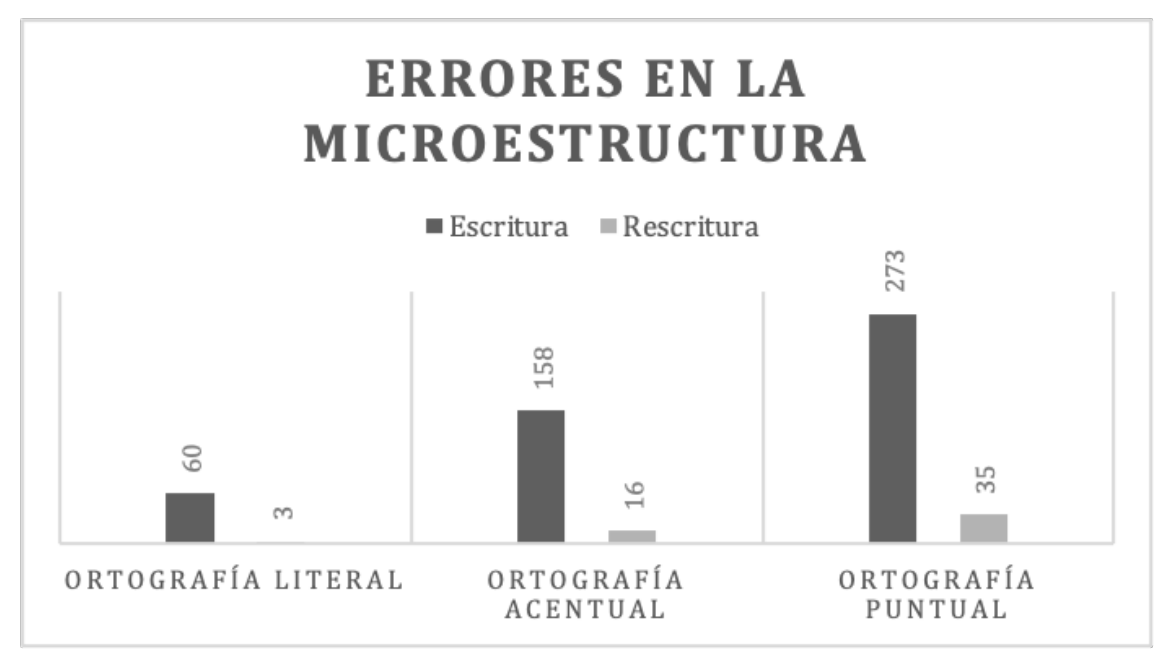

Gráfico 3. Errores en la microestructura.

La sumatoria de errores de la muestra da cuenta de la disminución significativa en las tres dimensiones del discurso: micro, macro y superestructura, puesto que de 830 se disminuyó a 77. Esto se asocia a los efectos de la retroalimentación correctiva de tipo indirecta.

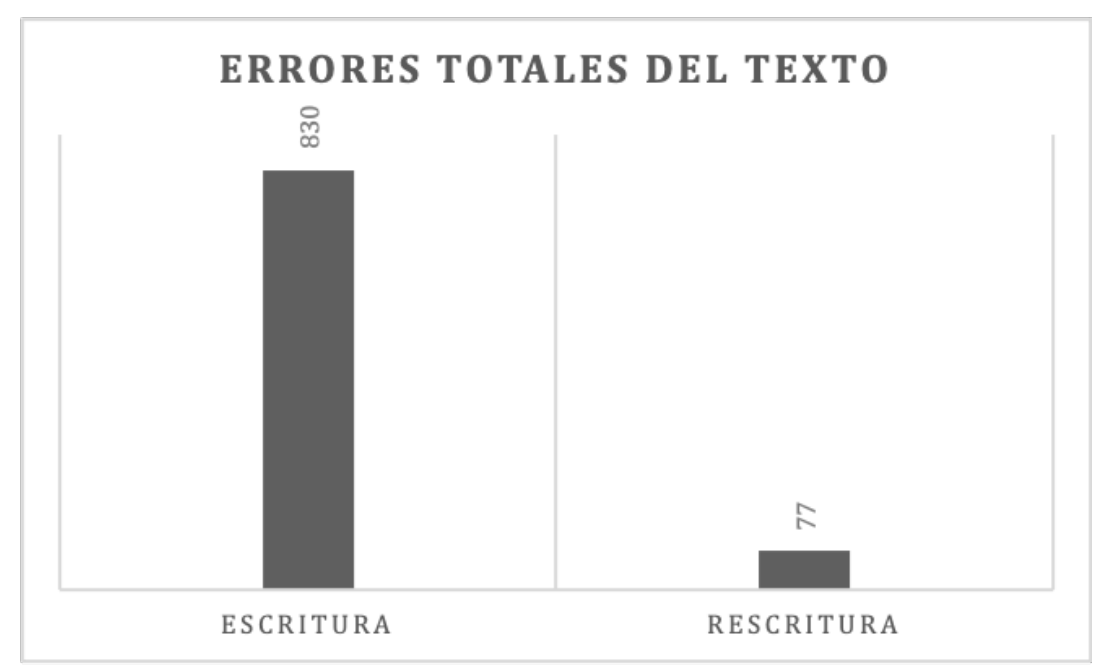

Gráfico 4. Sumatoria de errores. 
Artículo. Steffanie Kloss Medina, Angie Quintanilla Espinoza, Marcela Alexandre Moya. "La función de la retroalimentación correctiva indirecta en la reescritura de géneros académicos con modalidad narrativa elaborados por estudiantes novatos"

En cuanto a los criterios definidos para evaluar la calidad del escrito por los estudiantes la primera vez, la tabla $\mathrm{N}^{\circ} 1$ muestra la recurrencia de los errores.

\begin{tabular}{|l|l|l|l|l|l|l|l|l|l|}
\hline $\begin{array}{l}\text { Compon } \\
\text { Narrativo }\end{array}$ & $\begin{array}{l}\text { Estruc. } \\
\text { Narrat. }\end{array}$ & $\begin{array}{l}\text { Present. } \\
\text { del tema }\end{array}$ & $\begin{array}{l}\text { Propósito } \\
\text { comunicat }\end{array}$ & Coher. & Cohes & Voc & OL & OA & OP \\
\hline 28,00 & 29,00 & 28,00 & 16,00 & 50,00 & 83,00 & 105,00 & 60,00 & 158,00 & 273,00 \\
\hline
\end{tabular}

Tabla 4. Recurrencia de errores

En las primeras cuatro columnas, que expresan la idea de superestructura del texto, se presentan los fallos referentes al componente narrativo, estructura, tema y propósito. En estos datos se puede observar un bajo nivel de textos con problemas el estilo narrativo en comparación a los criterios restantes. En los tres primeros aspectos, los resultados fueron homogéneos. No obstante, en relación con el propósito, este solo tuvo 16 errores, es decir, de los 42 textos analizados, 16 presentaron problemas en ese descriptor, lo que se sostiene por las características del género con modalidad narrativa, pues este formato de escritura es más sencillo y conocido por los estudiantes, pues su voz se vislumbra a lo largo del escrito.

Ahora bien, si focalizamos en el quinto, sexto y séptimo descriptor, notamos que la concentración de errores es mayor que en la superestructura, puesto que los textos dan cuenta de problemas de coherencia, con 53 errores, de cohesión con 83, que se condice con los estudios del uso de marcadores del discurso y la dificultad que tienen los escritores para usarlos de manera efectiva (Kloss y Ferreira 185). Asimismo, el vocabulario es un indicador que también es elevado, ya que hay un total de 105 errores. El ámbito de la macroestructura textual es bastante complejo, entendiendo este nivel como el corazón del texto, debido a que aúna las ideas y da cuenta de la organización de los párrafos y la capacidad de los escritores de usar fórmulas lingüísticas adecuadas.

Para concluir, el último criterio de este trabajo remite a la ortografía, que aborda los descriptores ocho, nueve y diez. Los resultados en este ámbito muestran la dificultad que tienen los escritores al puntuar un escrito o tildar, según la normativa. En primer lugar, observamos que hay un total de 60 errores de ortografía literal, pero que este se agudiza en la ortografía acentual, en la que se contabilizaron 158 errores, lo que se incrementa mayormente en la puntuación del escrito, con un total de 273 errores. En síntesis, la 
sumatoria de la ortografía da un resultado de 491 errores, lo que concentra el 59,1\% de todos los errores cometidos.

El objetivo de este estudio considera revisar los errores más frecuentes en la escritura narrativa. No obstante, se entiende la limitación de una investigación de esa índole, por lo tanto, también se considera la reescritura del texto para notar si la revisión a través de la retroalimentación correctiva indirecta resulta eficiente en la superación de estos errores. Para revisar este apartado, la tabla 5 muestra los errores en la reescritura.

\begin{tabular}{|l|l|l|l|l|l|l|l|l|l|}
\hline $\begin{array}{l}\text { Componet } \\
\text { Narrativo }\end{array}$ & $\begin{array}{l}\text { Estruct. } \\
\text { Narrat. }\end{array}$ & $\begin{array}{l}\text { Presentación } \\
\text { del tema }\end{array}$ & $\begin{array}{l}\text { Propósito } \\
\text { comunicativo }\end{array}$ & Coher. & Cohes. & Voc & OL & OA & OP \\
\hline 4,00 & 4,00 & 2,00 &, 00 & 4,00 & 6,00 & 3,00 & 3,00 & 16,00 & 35,00 \\
\hline \multicolumn{8}{|c|}{ Tabla 5. Errores en la reescritura } \\
\hline
\end{tabular}

Lo que se observa en la tabla es la superación de los errores, pues de un total de 830 en todo el corpus y de 491 errores en ortografía, la reescritura solo muestra 77 formas erróneas. Cabe señalar, que la manifestación errónea se mantiene ligeramente más alta en la ortografía puntual. No obstante, la disminución sigue siendo significativa, ya que, de 273 errores en el uso de la puntuación, la segunda escritura solo refleja 35. Los resultados se condicen con las investigaciones de Tapia-Ladino et al. (3) que develó cómo los comentarios escritos gatillan una serie de acciones que dinamizan el proceso de escritura, en ese sentido, dan luces o lineamientos a los estudiantes a través del diálogo que se produce, lo que les que permite mejorar su proceso de atención y revisión de sus textos. Entonces, las retroalimentaciones se asocian a cierta mejora en los procesos de escritura, los que han quedado manifiestos en trabajos como los de Muñoz y Ferreira (84), Kloss y Ferreira (184) y Osle (2), cuyos énfasis han sido puestos en comparar estrategias de corrección y determinar la efectividad de la retroalimentación metalingüística e indirecta para que el sujeto piense y repare su error. Sin embargo, estos estudios, al trabajar con la etiqueta de feedback correctivo, solo focalizan en errores de lengua, descuidando detalles como el género y el propósito comunicativo inmersos en la retroalimentación.

Ahora bien, la tabla anterior también da cuenta de los aspectos superestructurales, tales como el componente narrativo, la estructura, la presentación del tema y el propósito. Lo que demuestra que, con una indicación detallada, los sujetos son capaces de mejorar a 
Artículo. Steffanie Kloss Medina, Angie Quintanilla Espinoza, Marcela Alexandre Moya. "La función de la retroalimentación correctiva indirecta en la reescritura de géneros académicos con modalidad narrativa elaborados por estudiantes novatos"

cabalidad la organización superestructural de su escrito. El primer borrador mostraba que, de un total de 42 textos, 28 tenían problemas en los componentes narrativos, 29 en la estructura narrativa, 28 en el tema y 16 en el propósito; estos resultados son totalmente opuestos en la reescritura, pues estructura y componentes solo presentaron 4 errores cada uno, mientras que hubo 2 errores en el tema y 0 en el propósito. Esto da cuenta de lo importante que es no solo centrar la corrección en la gramática y la ortografía, sino también en la organización retórica.

Otro aspecto relevante en este trabajo es revisar en la reescritura del texto si los estudiantes cometieron nuevos errores. Este punto es importante porque generalmente las investigaciones en el ámbito (Osle 2, Muñoz y Ferreira 84) se centran en revisar si el estudiante corrigió o no el error a partir de los comentarios del docente, pero poco se ha investigado respecto a los nuevos errores que surgen en el segundo escrito.

A continuación, la tabla 6 muestra los nuevos errores que cometieron los alumnos. Estos ascienden a un total de 232 errores nuevos.

\begin{tabular}{|l|l|l|l|l|l|l|l|}
\hline Coherencia & Cohesión & Vocabulario & OL & OA & OP & $\begin{array}{l}\text { Total } \\
\text { Ortografía }\end{array}$ & $\begin{array}{l}\text { Total } \\
\text { errores } \\
\text { Nuevos }\end{array}$ \\
\hline 10,00 & 20,00 & 28,00 & 33,00 & 43,00 & 98,00 & 174,00 & 232,00 \\
\hline
\end{tabular}

Tabla 6. Nuevos errores en la reescritura de textos narrativos

De acuerdo con lo observado en la tabla, podemos precisar que la mayor cantidad de errores se da en la ortografía puntual (98 errores), luego en la ortografía acentual (43) y finalmente en la ortografía literal (33). Estos resultados condicen no a nivel de cantidad, pero sí a nivel de categoría, con los datos obtenidos en la primera escritura, antes de recibir retroalimentación. Este aspecto es interesante de observar, pues, si bien los alumnos disminuyeron considerablemente sus errores de ortografía, aún no asimilan la normativa, ya que cometen nuevamente errores en este ámbito (Sotomayor et al. 322). En este sentido, la ortografía debiera llegar a automatizarse como parte de los procesos de codificación, lo cual permitiría utilizar la memoria de trabajo en otros procesos como la planificación o la revisión de la escritura (McCutchen 61). 
A raíz de ello, entendemos que los errores ortográficos se conciben como una habilidad básica en la escritura, puesto que se adquiere en los primeros años de escolarización. No obstante, si el dominio de habilidades cognitivas inferiores no se trabajó adecuadamente en estas etapas, podría ser un predictor de la adquisición del código escrito y según Berninger et al. (260) alcanzaría a influir en los procesos de transcripción y en la adquisición de habilidades cognitivas superiores de los procesos de composición en etapas futuras del desarrollo, lo que se condice con los resultados expuestos en este trabajo, pues los participantes, son estudiantes de primer año de universidad. Por lo tanto, para lograr este aprendizaje es necesario considerar a los alumnos como sujetos pensantes (Ferreiro 374), para potenciar una reflexión en torno a las normas ortográficas que permita generar conocimiento metacognitivo.

\section{Conclusiones}

En esta investigación, hemos respondido al objetivo principal, que hace referencia a la determinación de la frecuencia de errores en un texto especializado. Esto arrojó como resultado que los estudiantes cometían errores en diferentes niveles discursivos, es decir: microestructurales, macroestructurales y superestructurales. Sin embargo, estos errores son mejorados de manera considerable por el efecto de la retroalimentación correctiva indirecta, que coincide con los estudios en el ámbito.

En cuanto a los efectos de la retroalimentación, es interesante analizar este proceso, pues los resultados muestran mejoras significativas. No obstante, el aporte de este estudio es mostrar que sí se corrigen estos errores en los distintos niveles discursivos, pero que, en el ámbito de la microestructura, específicamente en la ortografía, los errores persisten y se materializan a través de nuevos errores. Esto no se manifiesta por la efectividad de la retroalimentación porque los sujetos mejoran a partir de las correcciones indirectas, pero comenten nuevos errores de ortografía puntual y acentual, lo que nos permite reflexionar sobre los reales efectos de la retroalimentación a largo plazo.

Los estudios en retroalimentación muestran su efectividad en los distintos contextos que se han aplicado. No obstante, incorporar el foco en el análisis de los nuevos errores 
Artículo. Steffanie Kloss Medina, Angie Quintanilla Espinoza, Marcela Alexandre Moya. "La función de la retroalimentación correctiva indirecta en la reescritura de géneros académicos con modalidad narrativa elaborados por estudiantes novatos"

cometidos por los escritores, nos admite inferir que tiene beneficios, aunque para que tenga efectos reales, este trabajo debe ser constante, hasta que los sujetos escritores sean capaces de internalizar las reglas de la gramática y operacionalizarlas en sus producciones escritas.

Sobre la base de los resultados de esta investigación, se plantean aportes pedagógicos vinculados a la efectividad de la retroalimentación correctiva indirecta. Esta estrategia metodológica es adecuada para mejorar la escritura de estudiantes novatos en un escenario de uso natural de la lengua en los tres niveles discursivos abordados en este estudio.

A nivel general, se puede concluir que es fundamental trabajar con la producción y corrección de textos en las distintas áreas, pues la llegada de un estudiante universitario a la disciplina que eligió para formarse académicamente, en palabras de Carlino (Escribir 2) es como la incursión de un inmigrante a una cultura nueva, con todas las características del visitar, y vivenciar valores y normas de comportamiento de la cultura de esa comunidad.

También, el trabajo con corpus resultó ser una metodología idónea para el análisis de errores, lo que admitió disponer de muestras reales de la lengua para tener claridad en los errores cometidos por los estudiantes y, posteriormente, establecer lineamientos para enseñar la escritura en contextos académicos.

Finalmente, las limitaciones de este estudio radican en la cantidad de textos analizados, pues para sacar resultados contundentes y con mayor significancia estadística, sería pertinente trabajar con mayor cantidad de textos y por un periodo más prolongado de tiempo. Finalmente, como proyección se puede establecer una comparación entre las disciplinas estudiadas para conocer la efectividad de la retroalimentación correctiva indirecta según la disciplina y en distintos géneros discursivos, pues la narración es esencial para el desarrollo de otras texturas discursivas, pero sería interesante replicar este estudio en géneros complejos como informes y ensayos académicos.

\section{REFERENCIAS}

Adam, Jean-Michel. “Types de séquences textuelles élémentaires”. Pratiques 56 (1987): 54-79. Impreso.

Ávila, Natalia, Federico Navarro y Mónica Tapia-Ladino. "Identidad, voz y agencia: Claves para una enseñanza inclusiva de la escritura en la universidad". Archivos Analíticos de Políticas Educativas, 4 (2020): 1-31. 
Berninger, Virginia, Cheryl Yates, Ana Cartwright, Judith Rutberg, Elizabeth Remy \& Robert Abbott (1992). Lower-level developmental skills in beginning writing. Reading and Writing, 260 (1992): 257-280. Impreso.

Brown, Douglas. Principles of Language Learning and Teaching. Englewood Cliffs, N.J: Prentice-Hall, 1994. Impreso.

Calle-Arango, Lina y Natalia Avila-Reyes. “Alfabetización académica chilena: revisión de investigaciones de una década". Literatura y lingüistica 41 (2020): 455-482. Impreso.

Carlino, Paula. "El proceso de escritura académica: cuatro dificultades de la enseñanza universitaria". Educere 3.26 (2004): 321-327. Impreso.

Carlino, Paula. Escribir, leer y aprender en la universidad. Una introducción a la alfabetización académica. Buenos Aires, Argentina: Fondo de Cultura Económica, 2005. Impreso.

Cassany, Daniel. Reparar la escritura. Didáctica de la corrección de lo escrito. Barcelona: Graó, 2014. Impreso.

Castelló, Montserrat. "De la investigación sobre el proceso de composición a la enseñanza de la escritura". Revista Signos 156 (2002): 149-162.

Chaudron, Craig. Second Language Classroom: Research on Teaching and Learning. New York: Cambridge University Press, 1988. Impreso.

Corder, Pit. "Idiosyncratic dialects and error analysis". IRAL 9.2 (1971): 147-160.

Córdova, Alejandro. "¿Qué es escribir para estudiantes ingresantes a la carrera de Ingeniería Civil? Un acercamiento a través de las representaciones sociales". Onomázein 30 (2015): 20-37. Impreso.

Cruz Piñol, Mar. Lingüística de corpus y enseñanza del español como 2/L. Madrid: Arco Libros, 2012. Impreso.

Ellis, Rod. "A typology of written corrective feedback types”. ELT journal 63.2 (2009): 97107. Impreso.

Ellis, Rod. "Researching the effects of form-focussed instruction on L2 acquisition". AILA Review 19 (2006): 18-41. Impreso.

Errázuriz, María. "El desarrollo de la escritura argumentativa académica: los marcadores discursivos". Onomázein 30 (2014): 217-236. Impreso.

Errázuriz, María et al. "Diagnóstico de la escritura de un ensayo de alumnos novatos de Pedagogía en el campus Villarrica UC, Chile”. Perfiles Educativos 37.150 (2015): 76-90. Impreso.

Ferreiro, Emilia. Alfabetización de niños y adultos: textos escogidos. Pátzcuaro, México: CREFAL.374 (2007):1-450. Impreso.

Flower, L. \& Hayes, J. “A Cognitive Process Theory of Writing”. College Composition and Comunication 369 (1981): 365-387. Impreso.

Hernández, Gerardo. "Teorías implícitas de escritura en estudiantes pertenecientes a dos comunidades académicas distintas". Perfiles Educativos 34.136 (2012): 42-62.

Hugo, Evelyn et al. "Intertextualidad manifiesta en textos de estudiantes universitarios. Caracterización de las citas en una etapa de formación académica inicial". Onomázein 41 (2018): 29-56. Impreso. 
Artículo. Steffanie Kloss Medina, Angie Quintanilla Espinoza, Marcela Alexandre Moya. "La función de la retroalimentación correctiva indirecta en la reescritura de géneros académicos con modalidad narrativa elaborados por estudiantes novatos"

Kotz, Gabriela y Anita Ferreira. "La precisión gramatical mediada por la tecnología: El análisis y tratamiento automático de errores”. Literatura y Lingüística 27 (2013): 219- 242. Impreso.

Kloss, Steffanie y Anita Ferreira. "La escritura de crónicas periodísticas informativas: una propuesta de avance desde el feedback correctivo escrito". Onomázein 184-185 (2019):161-185.

Kloss, Steffanie. "Análisis de errores: Una base teórica para mejorar la producción escrita de estudiantes de Periodismo". Tesis para optar al grado de Magister en Lingüística Aplicada, Universidad de Concepción, 2014. Impreso.

Mccutchen, Deborah. "From novice to expert: Implications of language skills and writingrelevant knowledge for memory during the development of writing skill". Journal of Research on Writing 61 (2011): 51-68. Impreso.

Muñoz, Belén y Anita Ferreira. "El feedback correctivo escrito indirecto en el aprendizaje de la forma comparativa de adjetivos en inglés". Logos 84 (2017): 73-89. Impreso.

Ortiz, Elsa. "La escritura académica universitaria: estado del arte". Ikala 16.28 (2011): 1741. Impreso.

Osle, Ángel. "Efectos del feedback directo e indirecto en el desarrollo de la competencia escrita de un grupo de estudiantes de español como lengua extranjera [Effects of direct and indirect written corrective feedback on students' written productions in a class of Spanish as a foreign language]". Lingüistica en la Red 2 (2019): 1-10.

Reyes, Graciela. Manual de redacción: Cómo escribir bien en español. Madrid: Arco Libros, 2008. Impreso.

Sheen, Younghee. Corrective Feedback Individual Differences and Second Language Learning. New York: Springster, 2011. Impreso.

Sotomayor, Carmen, Natalia Ávila, Percy Bedwell, Ana Domínguez, Gabriela Gómez y Elvira Jéldrez. "Desempeño ortográfico de estudiantes chilenos: claves para la enseñanza de la ortografía”. Estudios pedagógicos 322 (2017): 315-332.

Tapia-Ladino, Mónica, Beatriz Arancibia y Roxanna Correa. "Rol de los Comentarios Escritos en la Construcción de la tesis desde la perspectiva de estudiantes tesistas y profesores guías". Universitas Psychologica 3 (2016): 1-14.

Van Beuningen, Catherine. "Corrective Feedback in L2 Writing: Theoretical Perspectives, Empirical Insights, and Future Directions". International Journal of English Studies 10.2 (2010): 1-27.

Van Dijk, Teun y Walter Kintsch. Strategies of discourse comprehension. New York: Academic Press, 1983. Impreso.

Van Dijk, Teun. La ciencia del texto. Barcelona: Paidós, 1978. Impreso.

Vázquez, Graciela. ¿Errores? ;Sin falta! Madrid: Edelsa, 1999. Impreso. 